The effect of

\section{retrobulbar irradiation}

on exophthalmos, ductions and soft tissue signs in Graves'

ophthalmopathy: a retrospective analysis of 90 cases

\begin{abstract}
Purpose Retrospective analysis of the effect of retrobulbar irradiation on exophthalmos, ductions and soft tissue signs in patients with Graves' ophthalmopathy.

Methods We analysed the charts of 111 consecutive patients who were treated with retrobulbar irradiation according to standardised intake criteria between 1992 and 1997. After exclusion of patients who underwent other treatment (with steroids or orbital decompression) shortly before or within 6 months after irradiation, and on whom insufficient data were available, 90 patients were included. For these 90 patients, we analysed the exophthalmometry, ductions, soft tissue signs and visual acuity shortly before irradiation and after 3 and 6 months, respectively.

Results In the whole group, the Hertel value was on average $22 \mathrm{~mm}$ (SD 2.9) both before irradiation and after 3 and 6 months of followup. Separate analysis of data on 25 patients with bilateral exophthalmos of more than $24 \mathrm{~mm}$ also revealed no change in exophthalmos at followup. In the whole group, both abduction and elevation had improved by about $1^{\circ}$ (SD $6.6^{\circ}$; $p=0.05$ ) after 3 months. This improvement has little clinical significance. In a subgroup of 14 patients who showed more than $10^{\circ}$ of restricted eye motility in one or more directions, both abduction and elevation had increased by about $4^{\circ}\left(\mathrm{SD} 10^{\circ} ; p=0.02\right)$ at 3 and 6 months follow-up. Soft tissue signs had improved at 6 months after irradiation. We found no change in visual acuity after irradiation.

Conclusion Retrobulbar irradiation in Graves' ophthalmopathy does not seem to reduce exophthalmos. It probably improves eye motility in patients with severe restrictions. The late improvement in soft tissue signs may either be a late effect of irradiation or be related to the natural history of the disease.
\end{abstract}

Key words Graves' ophthalmopathy, Retrobulbar irradiation
R. L. J. VAN RUYVEN,

W.A. VAN DEN BOSCH,

P.G.H. MULDER,

W.M.H. EIJKENBOOM,

A.D.A. PARIDAENS
Graves' ophthalmopathy affects about $50 \%$ of dysthyroid patients. ${ }^{1}$ The disease is characterised by a panorbital inflammation which may lead to various degrees of exophthalmos, swelling of the eyelids and restriction of eye movements.

Because the disease is assumed to have an autoimmune aetiology, medical treatment aims at reducing the inflammatory reaction with immunosuppressive agents, such as prednisone or cyclosporine. ${ }^{2}$ Unfortunately, such treatment may be associated with considerable side-effects.

Retrobulbar irradiation as a means to suppress the orbital inflammation was introduced in $1914,{ }^{3}$ and has been widely applied since. The treatment consists of the administration of $20 \mathrm{~Gy}$ of irradiation retrobulbarly in daily fractions of $2 \mathrm{~Gy}$ within a 2 week period. ${ }^{4,5}$ In the literature, data on the results of retrobulbar irradiation are unclear and conflicting. While some authors have reported reduction of proptosis, others found improved eye motility or improved soft tissue signs. ${ }^{6-11}$

In this paper, we present the results of a retrospective analysis of the effect of retrobulbar irradiation on exophthalmos, ductions and soft tissue signs in 90 consecutive patients treated according to a standardised treatment protocol.

\section{Methods}

We reviewed the charts of 111 consecutive patients with Graves' ophthalmopathy who were treated with retrobulbar irradiation at our orbital service between 1992 and 1997.

According to our treatment protocol, retrobulbar irradiation was performed if the patient had been euthyroid for at least 3 months and showed no signs of 'malignant' Graves' ophthalmopathy (corneal ulceration or presumed optic nerve compression because of a diminished visual acuity or abnormal visually
R.L.J. van Ruyven

W.A. van den Bosch

A.D.A. Paridaens

Orbital Department

Rotterdam Eye Hospital

Rotterdam, The Netherlands

P.G.H. Mulder

Department of Medical

Biostatistics

Erasmus University

Rotterdam

Rotterdam, The Netherlands

W.M.H. Eijkenboom

Department of

Radiotherapy

University Hospital Dijkzigt

Rotterdam

Rotterdam, The Netherlands

A.D.A. Paridaens, MD, PhD The Rotterdam Eye Hospital Schiedamsevest 180

3011 BH Rotterdam

The Netherlands

e-mail: Paridaens@ned.net

Received: 15 December 1999 Accepted in revised form: 25 February 2000 
Table 1. Numerical transposition of the NOSPECS classification to facilitate the scoring of soft tissue signs

\begin{tabular}{ccc}
\hline Class & Description & Score \\
\hline 2 & Soft tissue involvement \\
o & None & 0 \\
a & Minimal & 1 \\
b & Moderate & 2 \\
c & Marked & 3 \\
3 & Exophthalmos & \\
o & $<23 \mathrm{~mm}$ & 0 \\
a & $23-24 \mathrm{~mm}$ & 1 \\
b & $25-27 \mathrm{~mm}$ & 2 \\
c & $>27 \mathrm{~mm}$ & 3 \\
4 & Extraocular muscle involvement \\
o & None & 0 \\
a & Limitation of motion in extremes of gaze \\
b & Evident restriction of motion & 1 \\
c & Fixation of the globe or globes & 2 \\
\hline
\end{tabular}

evoked potentials). Furthermore, the symptoms and signs had to have been present for less than 1 year, and the patient had to show at least one symptom or sign within the following NOSPECS classes: moderate or marked soft tissue signs (classes $2 b$ and $c$ ), uni- or bilateral exophthalmos of $23 \mathrm{~mm}$ or more (classes $3 \mathrm{a}, \mathrm{b}$ and c) and limitation of eye motility (classes $4 a, b$ and c) (Table 1).

Retrobulbar irradiation was performed according to the method described by Kriss ${ }^{4}$ and Donaldson et al. ${ }^{5} \mathrm{~A}$ cumulative dose of $20 \mathrm{~Gy}$ was administered to the retrobulbar space in 10 daily fractions of 2 Gy within a period of 2 weeks.

For retrospective analysis, patients were excluded in whom another intervention, such as orbital surgery or immunosuppressive treatment, had been performed within 3 months before or 6 months after irradiation. Using these criteria, 10 patients were excluded because orbital decompression had been performed. Two other patients had been treated with prednisone shortly before radiotherapy, 1 patient was lost to follow-up, and for 8 patients some data were lacking. Finally, 90 patients were included: 70 women $(78 \%$; mean age 47 years, SD 9.8 years) and 20 men (22\%; mean age 40.5 years, SD 9 years).

For these 90 patients, the exophthalmos, soft tissue signs, ductions and visual acuity were analysed. The exophthalmos had been assessed with a Hertel exophthalmometer, the symptoms and signs had been scored according to the NOSPECS classification. ${ }^{12}$ To facilitate analysis, we transposed the NOSPECS classification into a numerical system, in which the $a, b$ and c categories were arbitrarily given 1, 2 and 3 points respectively, as depicted in Table 1 . The ductions had been measured with a synoptophore in four directions of gaze at our orthoptic department. Normal ductions were defined as an abduction and adduction of more than $40^{\circ}$ and an elevation and depression of more than $30^{\circ}$. The visual acuity had been measured with a Snellen chart.

Statistical analysis was performed with the Wilcoxon paired samples test.

\section{Results}

The mean Hertel value before radiotherapy was $22 \mathrm{~mm}$ (range 13-31 mm, SD $2.8 \mathrm{~mm}$ ). At both 3 and 6 months follow-up the mean Hertel value remained $22 \mathrm{~mm}$ (range 14-31 mm, SD $2.8 \mathrm{~mm}$ ). Separate analysis of the exophthalmometry of 25 patients with a bilateral Hertel reading of more than $24 \mathrm{~mm}$ shortly before irradiation also showed no change of their exophthalmos at follow-up.

The measurements of the ductions showed an improvement of both abduction $(p=0.05)$ and elevation $(p=0.02)$ of about $1^{\circ}$ after 3 months. Virtually no further change occurred between 3 and 6 months follow-up (Fig. 1).
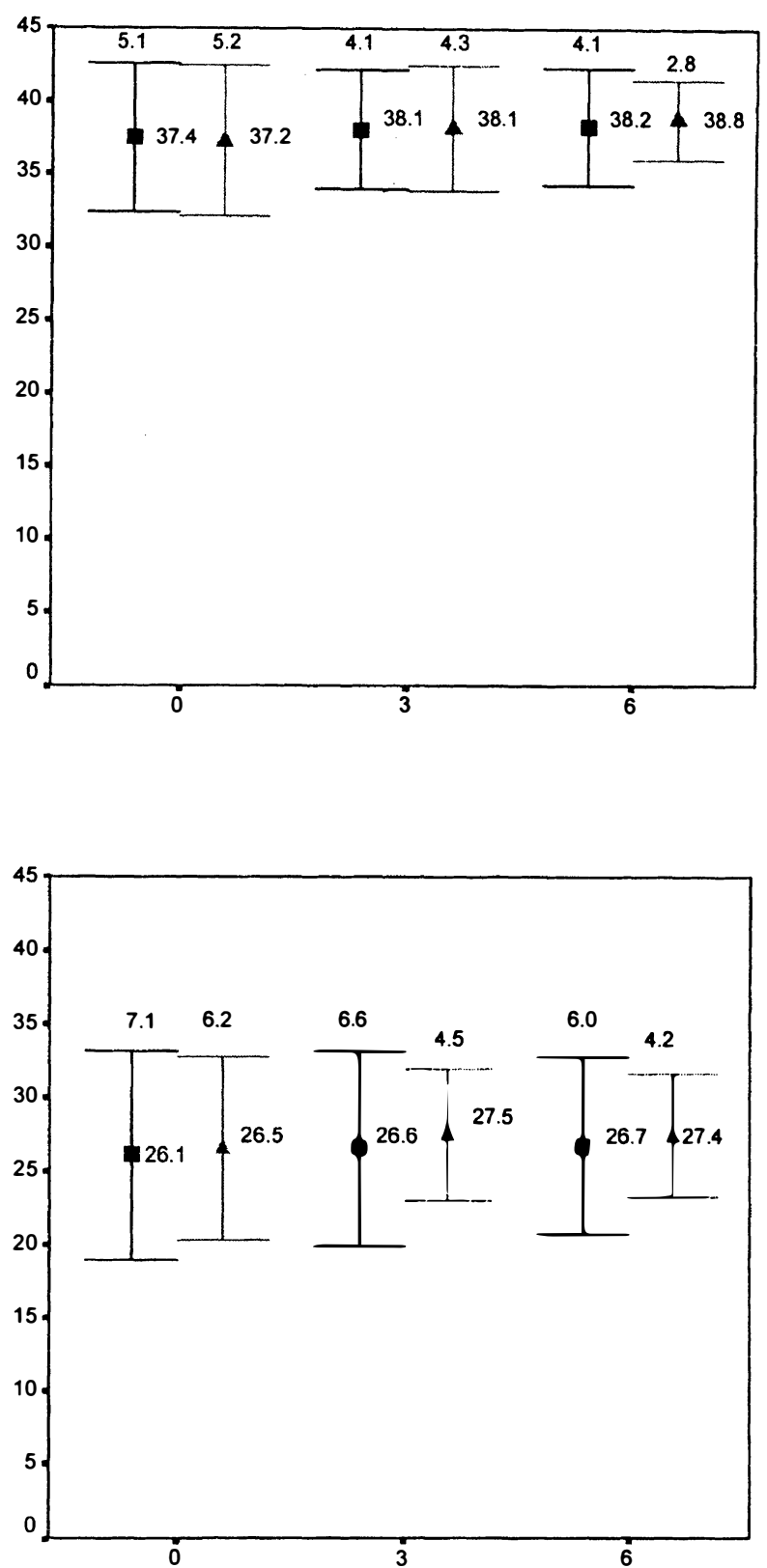

Fig. 1. Mean abduction (above) and elevation (below) with standard deviation of all patients. The horizontal axis shows the time in months after radiotherapy. The vertical axis shows duction in degrees. High-low bars represent the standard deviation. Squares, right eye; triangles, left eye. 

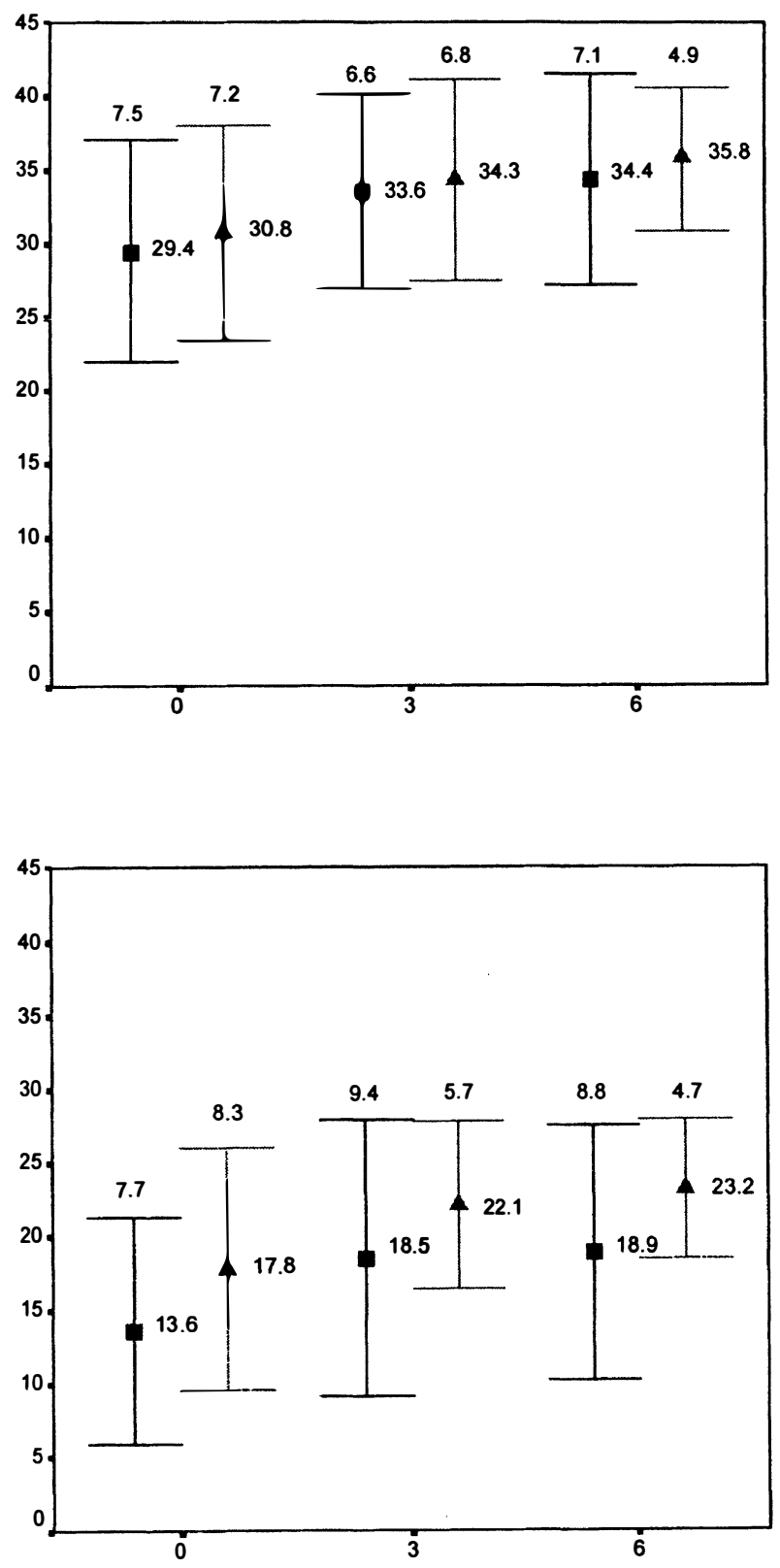

Fig. 2. Mean abduction (above) and elevation (below) with standard deviation of 14 patients with limited ductions of more than $10^{\circ}$. The horizontal axis shows the time in months after radiotherapy. The vertical axis shows duction in degrees. High-low bars represent the standard deviation. Squares, right eye; triangles, left eye.

We separately analysed the data of the 14 patients who showed a restricted motility of more than $10^{\circ}$ in one or more directions. After 3 months their mean abduction had improved by $4^{\circ}(p=0.009)$, and their mean elevation also by $4^{\circ}(p=0.02)$. Also in this group, we virtually found no further change at 6 months follow-up (Fig. 2).
The numerical analysis of the soft tissue signs showed an average score of 1.0 (SD 0.7) before irradiation and of 0.8 (SD $0.8, p=0.6$ ) at 3 months thereafter. At 6 months after irradiation the average score had improved to 0.6 (SD 0.6, $p=0.1$ ). Table 2 summarises the NOSPECS score. The visual acuity was on average 0.92 both before irradiation and at follow-up.

\section{Discussion}

Our data reveal no effect of retrobulbar irradiation on exophthalmos. Even in patients with a large amount of exophthalmos - in whom more effect might theoretically be expected - no reduction in exophthalmos could be detected. A reduction in exophthalmos after irradiation has been described by Petersen et al. However, their report was unclear regarding the amount of reduction achieved. ${ }^{13}$ Because of the retrospective character of our study, our data must be viewed with caution, since we cannot exclude the possibility that irradiation prevented an increase in exophthalmos. This, however, seems unlikely in view of the reported natural history of the disease. Perros et al. ${ }^{14}$ studied the course of ophthalmopathy in a euthyroid group who did not receive any immunosuppressive or surgical treatment. They found a spontaneous decrease in exophthalmos after 1-3 months.

After irradiation, both elevation and abduction improved. However, this average increase of about $1^{\circ}$ holds little - if any - clinical significance, in contrast with the marked improvement in ductions in the subset of patients with severely restricted motility at 3 months follow-up. In view of the generally protracted natural history of Graves' ophthalmopathy, it seems very likely that this improvement was due to the irradiation. We may therefore conclude that patients with severely restricted eye motility are likely to benefit from retrobulbar irradiation. Since the ductions showed virtually no further change between 3 and 6 months follow-up, we presume that the effect of irradiation on the extraocular muscles occurs within 3 months. Improvement of eye motility after irradiation has been reported by other authors, albeit in small groups of patients. $7,10,13$

An improvement in soft tissue signs was noted only after 6 months. In the above-mentioned follow-up study by Perros et al. ${ }^{14}$ spontaneous improvement in the soft tissue signs was noted after 3-6 months. Therefore, the improvement we found after irradiation cannot be differentiated from the natural history of the disease. ${ }^{14}$

Table 2. Average NOSPECS score, standard deviation and $\mathrm{p}$ value according to the classification system presented in Table 1, shortly before irradiation and at 3 and 6 months follow-up

\begin{tabular}{|c|c|c|c|c|c|c|c|c|c|}
\hline \multirow[b]{2}{*}{ Class } & \multirow[b]{2}{*}{ Description } & \multicolumn{2}{|c|}{ Before radiotherapy } & \multicolumn{2}{|c|}{3 months } & \multirow[b]{2}{*}{$p$ value } & \multicolumn{2}{|c|}{6 months } & \multirow[b]{2}{*}{$p$ value } \\
\hline & & Mean & $\mathrm{SD}$ & Mean & $\mathrm{SD}$ & & Mean & $\mathrm{SD}$ & \\
\hline 2 & Soft tissue signs & 1.0 & 0.7 & 0.8 & 0.8 & 0.6 & 0.6 & 0.6 & 0.01 \\
\hline 3 & Exophthalmos & 0.7 & 0.9 & 0.7 & 0.9 & 0.7 & 0.6 & 0.9 & 0.7 \\
\hline \multirow[t]{2}{*}{4} & Extraocular muscle involvement & 0.7 & 0.6 & 0.6 & 0.5 & 0.02 & 0.6 & 0.6 & 0.05 \\
\hline & Total score & 2.4 & & 2.1 & & & 1.8 & & \\
\hline
\end{tabular}


In conclusion, patients with severely restricted eye motility in Graves' ophthalmopathy probably benefit from retrobulbar irradiation. It does not seem to be indicated to treat exophthalmos. Because we excluded patients with signs of malignant Graves' ophthalmopathy and patients in whom another intervention was performed, we have no information on the effect of irradiation in this group. To better identify which patients might benefit from retrobulbar irradiation, a prospective randomised study is needed.

\section{References}

1. Jacobson DH, Gorman CA. Endocrine ophthalmopathy: current ideas concerning aetiology pathogenesis and treatment. Endocr Rev 1984;5:200-20.

2. Weetman AP, McGregor AM, Ludgate M, Beck L, Mills PV, Lazarus JH, et al. Cyclosporin improves Graves' ophthalmopathy. Lancet 1983;II:486-9.

3. Burch FE. The exophthalmos of Graves' disease. Minn Med 1929;12:668-76.

4. Kriss JP. Graves' ophthalmopathy: etiology and treatment. Hosp Pract 1975;10:125-33.

5. Donaldson SS, Bagshaw MA, Kriss JP. Supervoltage orbital radiotherapy for Graves' ophthalmopathy. J Clin Endocr Metab 1973;37:276-85.
6. Van Ouwerkerk BM, Wijngaarde R, Henneman G, Van Andel JG, Krenning EP. Radiotherapy of severe ophthalmic Graves' disease. J Endocrinol Invest 1985;8:241-7.

7. Prummel MF, Mourits MP, Blank L, Berghout A, Koornneef L, Wiersinga WM. Randomised double-blind trial of prednisone versus radiotherapy in Graves' ophthalmopathy. Lancet 1993;342:949-54.

8. Palmer D, Greenberg P, Cornell P, Parker RG. Radiation therapy for Graves' ophthalmopathy: a retrospective analysis. Int J Radiat Oncol Biol Phys 1987;13:1815-20.

9. Kao SC, Kendler DL, Nugent RA, Adler JS, Rootman J. Radiotherapy in the management of thyroid orbitopathy: computed tomography and clinical outcomes. Arch Ophthalmol 1993;111:819-23.

10. Hurbli T, Char D, Harris J, Weaver K, Greenspan F, Sheline G. Radiation therapy for thyroid eye disease. Am J Ophthalmol 1985;99:633-7.

11. Wilson WBW, Prochoda M. Radiotherapy for thyroid orbitopathy. Arch Ophthalmol 1995;113:1420-5.

12. Werner SC. Modification of the classification of the eye changes of Graves' disease. Am J Ophthalmol 1977;83:725-7.

13. Petersen IA, Kriss JP, McDougall IR, Donaldson SS. Prognostic factors in the radiotherapy of Graves' ophthalmopathy. Int J Radiat Oncol Biol Phys 1990;19:259-64.

14. Perros P, Crombie L, Kendall-Taylor P. Natural history of thyroid associated ophthalmopathy. Clin Endocrinol 1995;42:45-50. 